\title{
NECESSARY AND SUFFICIENT CONDITIONS FOR $L^{1}$ CONVERGENCE OF TRIGONOMETRIC SERIES
}

\author{
JOHN W. GARRETT AND ČASLAV V. STANOJEVIĆ
}

\begin{abstract}
It is shown that for the class of cosine series satisfying $a(n) \log n$ $=o(1)$ and $\Delta a(n)>0$ that integrability and $L^{1}$ convergence occur together. Relaxing the monotonicity to bounded variation we show that our previous result cannot be extended.
\end{abstract}

It is well known that the condition $a(n) \log n=o(1)$ is both necessary and sufficient for $L^{1}$ convergence for some classes of Fourier cosine series. Here we show, for the class of cosine series satisfying $a(n) \log n=o(1)$ and $\Delta a(n) \geqslant 0$, that integrability and $L^{1}$ convergence occur together. Relaxing the monotonicity to bounded variation we show that our previous result [1] cannot be extended. Finally we show that a cosine series with $\Delta a_{n} \geqslant 0$ is integrable if the norm of the derivative of the partial sums of its conjugate series are bounded.

In what follows $f(x)=\lim _{n \rightarrow \infty} S_{n}(x)$ where

$$
S_{n}(x)=\frac{1}{2} a(0)+\sum_{k=1}^{n}[a(k) \cos k x+b(k) \sin k x] .
$$

We denote $\sigma_{n}(x)=1 /(n+1) \sum_{k=0}^{n} S_{k}(x)$, and $\overline{S_{n}^{\prime}}(x)$ is the derivative of the conjugate of $S_{n}(x)$.

THEOREM 1. Let $a(n) \log n=o(1), b(n) \log n=o(1), \Delta a(n) \geqslant 0$, and $\Delta b(n)$ $\geqslant 0$. Then $\left\|\overline{S_{n}^{\prime}}\right\|=o(n)$.

Proof.

$$
\begin{array}{r}
\left\|\overline{S_{n}^{\prime}}\right\|=\left\|\sum_{k=1}^{n}[k a(k) \cos k x+k b(k) \sin k x]\right\| \\
=\| \sum_{k=1}^{n-1}\left\{[k \Delta a(k)-a(k+1)]\left[D_{k}(x)-\frac{1}{2}\right]\right. \\
\left.+[k \Delta b(k)-b(k+1)] \overline{D_{k}}(x)\right\} \\
+n a(n)\left[D_{n}(x)-\frac{1}{2}\right]+n b(n) \overline{D_{n}}(x) \|
\end{array}
$$

Received by the editors August 6, 1975.

AMS (MOS) subject classifications (1970). Primary 42A20, 42A32.

Key words and phrases. $L^{1}$ convergence of Fourier series, Fejér sums. 


$$
\begin{aligned}
\leqslant & B \sum_{k=1}^{n-1} k \Delta a(k) \log k+B \sum_{k=1}^{n-1} a(k+1) \log k \\
& +B \sum_{k=1}^{n-1} k \Delta b(k) \log k+B \sum_{k=1}^{n-1} b(k+1) \log k \\
& +B n a(n) \log n+B n b(n) \log n
\end{aligned}
$$

where $D_{n}(x)$ and $\overline{D_{n}}(x)$ are the Dirichlet and conjugate Dirichlet kernels, and $B$ is an absolute constant arising from the fact that

$$
\left\|D_{n}(x)-1 / 2\right\|=O(\log n) \text { and }\left\|\overline{D_{n}}(x)\right\|=O(\log n) .
$$

Four terms are $o(n)$ since $a(n) \log n=o(1), b(n) \log n=o(1)$, and the $(C, 1)$ method is regular. Thus,

$$
\begin{aligned}
\left\|\overline{S_{n}^{\prime}}\right\| \leqslant B & \sum_{k=1}^{n-1} k[\Delta a(k)+\Delta b(k)] \log k+o(n) \\
=B \sum_{k=1}^{n-1}\{k \Delta([a(k)+b(k)] \log k) & \quad+k[a(k+1)+b(k+1)] \log [(k+1) / k]\}+o(n) \\
= & B \sum_{k=1}^{n-1}[a(k)+b(k)] \log k-B(n-1)[a(n)+b(n)] \log n \\
& +B \sum_{k=1}^{n-1}[a(k+1)+b(k+1)] \log (1+1 / k)^{k}+o(n) \\
= & o(n)
\end{aligned}
$$

since

$$
[a(n)+b(n)] \log n=o(1),
$$

the $(C, 1)$ method is regular, and $\log (1+1 / k)^{k}$ converges to one.

Corollary 1. Let $a(n) \log n=o(1), b(n) \log n=o(1), \Delta a(n) \geqslant 0$, and $\Delta b(n) \geqslant 0$. Then $f$ is integrable if and only if $S_{n}$ converges to $f$ in $L^{1}$ metric.

Proof. "If": Obvious. "Only if": It is well known that if $f$ is integrable then $\sigma_{n}$ converges to $f$ in $L^{1}$ metric. Hence $\left\|S_{n}-f\right\| \leqslant\left\|S_{n}-\sigma_{n}\right\|+\left\|\sigma_{n}-f\right\|$. But $\left\|S_{n}-\sigma_{n}\right\|=1 /(n+1)\left\|\overline{S_{n}^{\prime}}\right\|=o(1)$.

The following propositions are now apparent.

Proposition 1. Let $f$ be integrable. Then $S_{n}$ converges to $f$ in $L^{1}$ metric if and only if $\left\|\overline{S_{n}^{\prime}}\right\|=o(n)$.

Proposition 2. Let $\left\|\overline{S_{n}^{\prime}}\right\|=o(n)$. Then $f$ is integrable if and only if $S_{n}$ converges to $f$ in $L^{1}$ metric.

Indeed, for any sequence, $A(n)$, the following proposition holds. 
Proposition 3. Let $A(n)$ be a sequence of positive numbers.

(1) Let $\left\|\sigma_{n}-f\right\|=o(A(n))$. Then $\left\|S_{n}-f\right\|=o(A(n))$ if and only if $\left\|\overline{S_{n}^{\prime}}\right\|$ $=o(n A(n))$.

(2) Let $\left\|S_{n}-f\right\|=o(A(n))$. Then $\left\|\sigma_{n}-f\right\|=o(A(n))$ if and only if $\left\|\overline{S_{n}^{\prime}}\right\|$ $=o(n A(n))$.

(3) Let $\left\|\overline{S_{n}^{\prime}}\right\|=o(n A(n))$. Then $\left\|\sigma_{n}-f\right\|=o(A(n))$ if and only if $\left\|S_{n}-f\right\|$ $=o(A(n))$.

It is clear that Proposition 3 contains Proposition 1 as the special case where $A(n)=1$. Also, since $\left\|\overline{S_{n}^{\prime}}\right\|=o(1)$ is equivalent to $f$ being constant, we have the following special case. Let $n\left\|S_{n}-f\right\|=o(1)\left[n\left\|\sigma_{n}-f\right\|=o(1)\right]$. Then $n\left\|\sigma_{n}-f\right\|=o(1)\left[n\left\|S_{n}-f\right\|=o(1)\right]$ if and only if $f$ is constant.

In Corollary 1 we required $\Delta a(n) \geqslant 0$. Several results on $L^{1}$ convergence of cosine series are known that only require bounded variation of $a(n)$, that is, $\sum_{n=1}^{\infty}|\Delta a(n)|<\infty$. It is well known that if $a(n)=o(1)$ and $a(n)$ is quasiconvex $\left(\sum_{n=1}^{\infty}(n+1)\left|\Delta^{2} a(n)\right|<\infty\right)$ that $S_{n}$ converges to $f$ in $L^{1}$ metric if and only if $a(n) \log n=o(1)$. Using an inequality of Sidon, Telyakovskii [2] has proved the following theorem where quasi-convexity is relaxed.

TheOREM A. Let $f(x)=\lim _{n \rightarrow \infty} S_{n}(x)$ where $b(n)=0$ and $a(n)=o(1)$. Let numbers $A(n)$ exist such that $\Delta A(n) \geqslant 0, \sum_{n=0}^{\infty} A(n)<\infty$, and $|\Delta a(n)|$ $\leqslant A(n)$ for all $n$. Then $S_{n}$ converges to $f$ in $L^{1}$ metric if and only if $a(n) \log n$ $=o(1)$.

Recently we [1] found a condition necessary and sufficient for a modification of $S_{n}$ to converge to $f$ in $L^{1}$ metric.

THEOREM B. Let

$$
g_{n}(x)=\frac{1}{2} \sum_{k=0}^{n} \Delta a(k)+\sum_{k=1}^{n} \sum_{j=k}^{n} \Delta a(j) \cos k x,
$$

$b(n)=0, a(n)=o(1)$, and $\sum_{n=1}^{\infty}|\Delta a(n)|<\infty$. Then $g_{n}$ converges to $f$ in $L^{1}$ metric if and only if

$$
\text { for } \varepsilon>0 \text { there exists } \delta>0 \text { (independent of } n \text { ) such that }
$$

$$
\int_{0}^{\delta}\left|\sum_{k=n}^{\infty} \Delta a(k) D_{k}(x)\right|<\varepsilon .
$$

As a corollary we extended Telyakovskii's result.

Corollary B. Let $b(n)=0, a(n)=o(1), \sum_{n=1}^{\infty}|\Delta a(n)|<\infty$, and (C) be satisfied. Then $S_{n}$ converges to $f$ in $L^{1}$ metric if and only if $a(n) \log n=o(1)$.

Here we show that if we require the conditions $a(n)=o(1)$ and $\sum_{n=1}^{\infty}|\Delta a(n)|<\infty$ then Theorem A cannot be extended beyond Corollary B.

THEOREM 2. Let $b(n)=0, a(n)=o(1), \sum_{n=1}^{\infty}|\Delta a(n)|<\infty$, and $a(n) \log n$ $=o(1)$. Then $S_{n}$ converges to $f$ in $L^{1}$ metric if and only if condition (C) is satisfied.

Proof. Using $g_{n}$ as defined in Theorem B, 


$$
\begin{aligned}
\left\|S_{n}(x)-f(x)\right\|=\left\|\frac{1}{2} a(0)+\sum_{k=1}^{n} a(k) \cos k x-f(x)\right\| \\
=\| \frac{1}{2} a(0)-\frac{1}{2} a(n+1)+\sum_{k=1}^{n}[a(k)-a(n+1)] \cos k x-f(x) \\
\quad+\frac{1}{2} a(n+1)+\sum_{k=1}^{n} a(n+1) \cos k x\|\| \\
=\left\|\frac{1}{2} \sum_{k=0}^{n} \Delta a(k)+\sum_{k=1}^{n} \sum_{j=k}^{n} \Delta a(j) \cos k x-f(x)+a(n+1) D_{n}(x)\right\| \\
=\left\|g_{n}(x)-f(x)+a(n+1) D_{n}(x)\right\| .
\end{aligned}
$$

But $\left\|a(n+1) D_{n}(x)\right\|=o(1)$, since $a(n) \log n=o(1)$ and $\left\|D_{n}(x)\right\|$ $=O(\log n)$. Thus, $S_{n}$ converges to $f$ in $L^{1}$ metric if and only if $g_{n}$ converges to $f$ in $L^{1}$ metric. We see that the coefficients $a(n)$ satisfy the requirements of Theorem B, so the result follows.

At this point we see that if $\left|\sum_{n=1}^{\infty} b(n)\right|<\infty$ then $\left\|\overline{S_{n}}\right\|=O\left(\left\|\overline{S_{n}^{\prime}}\right\|\right)$. For

$$
\begin{aligned}
\left\|\overline{S_{n}}\right\| & =\int_{-\pi}^{\pi}\left|\int_{0}^{x} \overline{S_{n}^{\prime}}(t) d t+\sum_{n=1}^{\infty} b(n)\right| d x \\
& \leqslant \int_{-\pi}^{\pi} \int_{-\pi}^{\pi}\left|\overline{S_{n}^{\prime}}(t)\right| d t d x+2 \pi\left|\sum_{n=1}^{\infty} b(n)\right| \\
& =2 \pi\left\|\overline{S_{n}^{\prime}}\right\|+2 \pi\left|\sum_{n=1}^{\infty} b(n)\right| .
\end{aligned}
$$

This leads to integrability conditions for $f$ and $\bar{f}$, the conjugate of $f$.

Proposition 4. Let $\left|\sum_{n=1}^{\infty} b(n)\right|<\infty$. If $\left\|\overline{S_{n}^{\prime}}\right\|=O(1)$ then $\bar{f} \in L^{1}$. If in addition we require $\Delta a(n) \geqslant 0, \Delta b(n) \geqslant 0$, then $f \in L^{1}$.

\section{REFERENCES}

1. J. W. Garrett and Č. V. Stanojević, On $L^{1}$ convergence of certain cosine sums, Proc. Amer. Math. Soc. 54 (1976), 101-105.

2. S. A. Teljakovskii, Concerning a sufficient condition of Sidon for the integrability of trigonometric series, Mat. Zametki 14 (1973), 317-328 = Math. Notes 14 (1973), 742-748 (1974). MR 48 \#6798.

Department of Mathematics, University of New Orleans, New Orleans, louisiana 70122

Department of Mathematics, University of Missouri, Rolla, Missouri 65401 